\title{
Comparing two treatment approaches for patients with type 1 diabetes during aerobic exercise: a randomised, crossover study
}

\section{Varun Vartak}

University of Waikato

Lynne Chepulis ( $\square$ lynnec@waikato.ac.nz)

University of Waikato https://orcid.org/0000-0002-9661-4669

Matt Driller

La Trobe University

Ryan Paul

Waikato District Health Board

\section{Short communication}

Keywords: hypoglycaemia, hyperglycaemia, glycaemic control, insulin, carbohydrates

Posted Date: November 5th, 2020

DOI: https://doi.org/10.21203/rs.3.rs-33528/v2

License: (c) (i) This work is licensed under a Creative Commons Attribution 4.0 International License. Read Full License 


\section{Abstract}

In a randomised, counterbalanced, crossover design, eight men with type 1 diabetes (T1D; mean \pm SD age: $27.6 \pm 11.4$ years) reduced insulin (INS) by $50 \%$ of their normal dose or consumed carbohydrates equivalent to $1 \mathrm{~g}$ of carbohydrate/ $\mathrm{kg}$ of their body weight without the usual insulin bolus (CARBS) over two sessions, held a week apart. Each session included standardised meals, a 45-minute treadmill-walk at $7.24 \mathrm{~km} \cdot \mathrm{h}^{-1}$ and a six-minute walk test (6MWT). Rate of perceived exertion (RPE), blood glucose, blood ketone and blood lactate measures were taken before, during and immediately after the aerobic exercise. The distance covered in metres and the predicted $\mathrm{VO}_{2} \max \left(\mathrm{mL} \cdot \mathrm{kg}^{-1} \cdot \mathrm{min}^{-1}\right)$ were also calculated for the $6 \mathrm{MWT}$. Participants completing the INS intervention spent more time in normoglycaemia $(242 \pm 135 \mathrm{~min}$ vs $88 \pm 132 \mathrm{~min} ; P<0.01)$ and less time in hyperglycaemia ( $41 \pm 95 \mathrm{~min}$ vs $154 \pm 125 \mathrm{~min} ; P=0.01)$ as compared to the CARBS intervention. Mild hypoglycaemia occurred in two participants during INS and no participants during CARBS. Furthermore, there was no significant difference for blood lactate, ketone, $\mathrm{RPE}$, distance covered and predicted $\mathrm{VO}_{2}$ max between interventions. Based on this pilot study, INS intervention appears to be the best approach for maintaining blood glucose levels in those with T1D during aerobic exercise, though this does need evaluating in other groups, including women, children and those with sub-optimal glycaemic control.

Trial registration: ACTRN12619001397101p. Registered 09 September 2019, http://anzctr.org.au/Trial/Registration/TrialReview.aspx?id=378264

\section{Key Points}

- Reducing insulin appears to be more effective than eating carbohydrates without the usual insulin bolus for maintaining glycaemic control during aerobic exercise.

- Reduced insulin was the preferred intervention for $75 \%$ of participants when evaluated using a questionnaire with a closed-ended question (which intervention, if either, would you prefer for future exercise?).

\section{Introduction}

Exercise is a cornerstone of management of type 1 diabetes (T1D) as it can aid with glycaemic control, reduce cardiovascular disease and improve quality of life [1-3]. However, many patients with T1D are concerned about undertaking exercise because of the risk of developing hypoglycaemia and how to adjust their insulin or carbohydrate intake to prevent aberrations in their glycaemic control [3, 4]. Indeed, in a systematic review of 10 studies, the effects of physical exercise and recovery in T1D were shown to be dependent on exercise scheduling, duration and intensity of the exercise, prior carbohydrate consumption, insulin therapy, pre-exercise glucose levels, and cardiovascular fitness [5]. However, with an 
adequate knowledge of metabolic responses and appropriate diabetes self-management, patients with T1D should be able overcome their fear of exercise-induced dysglycaemia $[3,4]$.

Guidelines recommend that to prevent exercise-induced hypoglycaemia, patients with T1D must either reduce their basal insulin doses or consume carbohydrates without co-administration of insulin prior to commencing exercise [6, 7]. However, whilst research does indicate that both strategies can independently support glycaemic control during exercise [8]. Knowledge of whether one strategy is better than the other is lacking, and only limited peer-reviewed studies have compared both approaches during exercise within the same experimental design [9-11]. Thus, the aim of the current study was to evaluate the effects of these two approaches on glycaemic control (time in normo-, hypo- and hyper-glycaemia) and performance in a single bout of aerobic exercise. We also measured the effects of the two interventions on predicted $\mathrm{VO}_{2}$ max in a six-minute maximum walk test (6MWT), and on patient-perceived performance.

\section{Methods}

\section{Participants.}

Men with T1D were recruited directly from the Waikato Regional Diabetes Service (WRDS, Hamilton, New Zealand) between September and November 2019. To be eligible, all participants needed to have had T1D for at least two years, be aged 18 - 60 years, have a recent HbA1c (within the last 3 months) of 45 $90 \mathrm{mmol} / \mathrm{mol}$ (6.3 - 13.9\%); be able to walk/run unaided for 45 minutes at a speed of $7.24 \mathrm{~km} . \mathrm{h}^{-1}$ and be fully competent with diabetes self-management. Participants were excluded if they were taking testosterone therapy, had any comorbidities that would impact on their ability to complete the study (e.g. cardiovascular disease, cerebrovascular disease, severe diabetic retinopathy, nephropathy, neuropathy); were taking medications that altered heart rate (e.g. beta-blockers); or were currently using closed-loop insulin pump therapy. All potential participants were screened at the WRDS Clinic and upon recruitment were required to attend the clinic again 38-48 hours prior to the first test session to have a Flash Glucose monitoring (FGM) device (Abbott Freestyle Libre, Chicago, IL) inserted to allow for continuous glucose monitoring [12]. All participants were able to wear the Freestyle Libre device for 14 days from the date of insertion. The study was successfully registered under the Australia New Zealand Clinical Trial Registry (ACTRN12619001397101p) and was approved by the New Zealand Health and Disability Ethics Committee (ref 19/NTB/175).

\section{Study Design:}

This study was a randomised, counterbalanced, crossover design, in which men with T1D evaluated two different interventions for managing glycaemia whilst undertaking a pre-defined exercise protocol. The two sessions were carried out a week apart at the University of Waikato gymnasium in a controlled, indoor environment with meals (breakfast and lunch) provided. The sessions were conducted by a research team consisting of a diabetologist/endocrinologist, a clinical research specialist, a clinical nurse, 
a sport physiologist and a health research student. The night before each session, participants were required to consume a meal that contained at least $1 \mathrm{~g}$ of carbohydrate/ $\mathrm{kg}$ of body weight [6], and they were asked to eat the same meal before both intervention sessions. Consumption of alcohol, caffeinebased beverages and performing strenuous exercise were prohibited during the 12 hours before each aerobic exercise session. Participants then fasted following dinner on the previous day until 9am the following morning.

The two interventions assessed were i) reducing their insulin (INS) or ii) consuming carbohydrates without the usual insulin bolus (CARBS)) along with the breakfast meal. The same breakfast meal was provided at 9 am in both groups, and consisted of bananas, apple juice and $100 \%$ Isolate protein powder (Musclepharm COMBAT, New Zealand), with quantities individualised to contain $0.66 \mathrm{~g}$ of carbohydrate/kg of body weight and $3.375 \mathrm{kcal} / \mathrm{kg}$ of body weight, meeting both the requirements of exercise carbohydrates guidelines [6] and calculated energy expenditure using the Browning Walking Metabolic Prediction equation [13]. Dietitian advice was sought to ensure all meals, including the meal the night before the exercise, met the required recommendations. The accurate intake of breakfast was observed by the investigators and all participants confirmed that they had consumed the recommended meal the night prior.

Participants were either on multiple daily injections of insulin (MDI) or continuous subcutaneous infusion of insulin (CSIl; insulin pump therapy). All patients on MDI were on a long-acting basal insulin (insulin glargine) and rapid-acting bolus insulin (either insulin aspart or insulin lispro). All patients on insulin pump therapy used a continuous infusion of insulin aspart. During the INS intervention, participants on MDI did not alter their basal insulin, but halved their bolus insulin of their normal dose immediately before consuming the meal as a single bolus as per the consensus guidelines [7]. Those participants in the INS intervention on CSII started a 50\% temporary basal rate reduction 90 minutes before aerobic exercise and halved their normal bolus dose for their meal. The $50 \%$ temporary basal rate reduction stopped 60 minutes after completion of the aerobic exercise [7]. The participants in the CARBS intervention consumed their breakfast meal without the administration of any bolus insulin and did not alter their basal insulin [6]. Throughout the test sessions the participants were not allowed to further adjust their insulin doses or carbohydrates intake. Any adjustments (required or accidental) would trigger the cessation of the session for this participant.

All participants began the aerobic exercise at 10.00 am and were required to walk on a motorised treadmill (Life Fitness, Chicago, IL; USA) at a fixed speed of $7.24 \mathrm{~km} \cdot \mathrm{h}^{-1}$ (4.5 miles per hour) at a $1 \%$ gradient for 45 minutes. According to the ACSM classification, this would be classified as "vigorous" intensity exercise. Ten minutes following completion of the treadmill walk, participants then performed the 6MWT [14] by walking as quickly as possible for six minutes around the perimeter of an inside arena. This test is considered as a validated measure of predicted VO2 max, and this was calculated using the equation as follows [15]. $\mathrm{VO}_{2} \max \left(\mathrm{mL} \cdot \mathrm{kg}^{-1} \cdot \mathrm{min}^{-1}\right)=70.161+(0.023 \times$ six-minute walk distance covered $[\mathrm{m}])-(0.276 \times$ weight $[\mathrm{kg}])-(6.79 \times$ sex, where male $=0$, female $=1)-(0.193 \times$ resting heart rate [BPM] $)$ - $(0.191 \times$ age [years] $)$. For the $6 \mathrm{MWT}$, distances were marked off every $3 \mathrm{~m}$, and the total distance walked 
in the six minutes was recorded. Approximately sixty minutes after completion of the exercise activities, participants had lunch (One Square Meal, OSM: Queenstown, New Zealand), individualised to consist of 1 $\mathrm{g}$ of carbohydrate/kg of body weight and administered their normal dose of bolus insulin [7]. Water was allowed ad libitum throughout each session and no additional adjustments (including self-adjustment) of insulin or carbohydrate were allowed until three hours after lunch.

Capillary blood glucose was initially measured before breakfast. Capillary glucose and lactate were measured immediately at the start of the 45-minute aerobic exercise, every 15 minutes during the aerobic exercise, at the end of the 45-minute aerobic exercise, within 1-3 minutes after completion of the 6MWT [16] and before lunch. Blood ketone level measurements were taken at the beginning and the end of the 45-minute aerobic exercise and within 1-3 minutes after completion of the 6MWT. Blood lactate was measured using a lactate analyser (Lactate Pro 2, Arkray, Japan). Glucose levels were monitored directly using the FGM device, and also via capillary sampling (Abbott Optium glucose strips) to minimise any delay between the interstitial and blood glucose levels during physical activity [12]. Self-perceived rate of exertion (RPE) was also assessed every 15 minutes during the aerobic exercise, and immediately following the 6MWT using the Borg's RPE scale [17]. After completion of both sessions', participants were asked which intervention, if either, they preferred for future exercise.

\section{Statistical Analysis:}

Descriptive statistics and data are expressed as mean \pm standard deviation (SD). Paired T-tests (twotailed) were performed on blood glucose levels, blood ketone, blood lactate, RPE, distance covered and the predicted $\mathrm{VO}_{2}$ max for the two interventions. Repeated measures ANOVA was performed to compare time in the normal blood glucose range (primary outcome), time in hyperglycaemia and time in hypoglycaemia for INS and CARBS. Thresholds for normal range or normoglycaemia were $3.9-10$ $\mathrm{mmol} / \mathrm{L}$; mild hyperglycaemia between 10.1 - $13.9 \mathrm{mmol} / \mathrm{L}$; severe hyperglycaemia $\geq 14 \mathrm{mmol} / \mathrm{L}$; mild hypoglycaemia $<3.9 \mathrm{mmol} / \mathrm{L}$ and significant hypoglycaemia $(<3 \mathrm{mmol} / \mathrm{L})$. The time in each range was calculated from the FGM but importantly there were no significant differences or lag between capillary and interstitial glucose levels in any participants. Statistical significance was accepted at a level of $\mathrm{P}<$ 0.05 .

\section{Results}

Eight men with T1D participated in the study (age: $27.6 \pm 11.4$ years; weight: $91.8 \pm 11.0 \mathrm{Kg}$; BMl: $26.6 \pm$ $2.2 \mathrm{~kg} \cdot \mathrm{m}^{2}$; HbA1 c: $55.1 \pm 7.4 \mathrm{mmol} / \mathrm{mol} ; 7.2 \%$ ) and all were able to complete both sessions without the need for additional insulin correction. Six participants were on multiple daily injections of insulin (MDI) and two participants on CSII.

Overall, participants completing the INS intervention spent more time in normoglycaemia $(242 \pm 135$ min vs $88 \pm 132 \mathrm{~min} ; \mathrm{P}<0.01)$ and less time in severe hyperglycaemia (41 $\pm 95 \mathrm{~min}$ vs $154 \pm 125 \mathrm{~min} ; \mathrm{P}=$ 0.01 ) compared to the CARBS intervention (Figure 1). The time in mild hyperglycaemia was also halved 
during the INS intervention (mean $66 \pm 76 \mathrm{~min}$ vs $133 \pm 111 \mathrm{~min}$ ), though this was not statistically different $(P=0.23)$. Two participants out of the total eight participants (i.e. $25 \%)$ experienced mild hypoglycaemia during INS and no hypoglycaemia was observed in any participants during CARBS (26 \pm 45 min vs 0 min; $P=0.17$ ). Mean blood glucose levels remained significantly higher during CARBS than INS from 15 min into the aerobic exercise until the duration of the study, despite a comparable blood glucose level just prior to breakfast (Figure 1). From the repeated measures ANOVA, there was a statistically significant interaction between the two interventions (INS and CARBS) and time spent in each blood glucose level range $F(3,18)=6.574, \mathrm{P}<0.001$.

There were no significant differences in blood lactate, ketone levels or rate of perceived exertion (RPE) during the treadmill test at any timepoints between interventions (all $P>0.05$ ). However, participants completing INS intervention were likely able to walk further in the 6MWT (mean \pm SD $777.9 \pm 319.9 \mathrm{~m}$ vs

$724.6 \pm 302.2 \mathrm{~m}, \mathrm{P}=0.07$ ) with no change in predicted $\mathrm{VO}_{2} \max$ (mean $\pm \mathrm{SD} 39.7 \pm 16.2 \mathrm{~m}$ vs $38.8 \pm 16.0$ $\mathrm{m}, \mathrm{P}=0.38$ ) compared to the CARBS intervention. Self-perceived data indicated that $75 \%$ of participants preferred the INS intervention and would opt to use this strategy for future exercise.

\section{Discussion}

Only limited studies have evaluated the effects of both reducing insulin and eating carbohydrates without the usual insulin bolus on glycaemic control and performance with aerobic exercise [7, 9-11]. Here we compare these two recommendations and demonstrate that reducing insulin prior to exercising is the best strategy for maintaining normoglycaemia and likely the better strategy for optimising aerobic performance in patients with T1D. Furthermore, reducing insulin by $50 \%$ did not increase the risk of ketoacidosis or lactatemia. Others have also shown that reducing insulin is the best strategy for preventing hyperglycaemia $[8,18]$, but that consuming extra carbohydrates is the safest option in preventing hypoglycaemia $[10,19]$. However, as in this study, athletes with diabetes often prefer the risk of mild hypoglycaemia than hyperglycaemia due to the deleterious effects of the latter on exercise performance [20]. Nevertheless, it is clear that patients with T1D need individualised reductions in their insulin around exercise and the priority in the majority of patients will be preventing hypoglycaemia rather than performance [7]. In particular, patients that are keen to administer insulin around exercise need to ensure adequate monitoring of their glucose levels and have appropriate treatment for hypoglycaemia readily accessible.

Despite only being a small pilot study, strengths of this study include that participants acted as their own controls in a direct comparison of the two main approaches in T1D, and that unlike other studies, both groups had similar glucose levels at baseline. However, this study did not include women (to minimise the effects of the sex hormones), children or the elderly, and further larger studies are required in both male and female patients across the life span. Limitations of the current study and areas for future research include the implementation of a control trial using a typical day without additional carbohydrate consumption or insulin reduction, and also the use of a validated questionnaire to evaluate perceived performance and preference. All participants in this study also had good glycaemic control, so it would be 
worthwhile comparing these guideline recommendations in patients with suboptimal glycaemic control, because in many of these patients' reductions in insulin or extra carbohydrates may not be required, particularly with short periods of exercise. Lastly, the recommendations will likely differ depending on the different types, intensity and length of exercise.

\section{Declarations}

\section{Ethics Approval and Consent to Participate:}

This study was approved by the New Zealand Health and Disability Ethics Committee (HDEC) (ref 19/NTB/175) and conforms to the Declaration of Helsinki. Prior to participation, written informed consent was obtained.

\section{Consent for Publication:}

All authors have reviewed the manuscript and approved it for publication. No individual data is presented in this manuscript.

Availability of data and materials: The datasets used and/or analysed during the current study are available from the corresponding author on reasonable request.

Competing Interests: The authors declare that they have no competing interests.

Funding: This study was funded by the Waikato Regional Diabetes Service, Waikato District Health Board, Hamilton, New Zealand.

Author Contributions: RP, LC and MD designed the study. All authors were involved in the collection of data. VV analysed the data, and LC, RP and VV prepared the manuscript for publication. All authors approve the final manuscript.

Acknowledgements: We would like to thank the University of Waikato for providing a student scholarship to support VV during this study.

\section{List Of Abbreviations}

T1D Type 1 Diabetes

6MWT 6 Minute Walk Test

INS Reduced Insulin Intervention

CARBS Additional carbohydrates intervention

CSII Continuous subcutaneous infusion of insulin 


$\begin{array}{ll}\mathrm{VO}_{2} \text { max } & \text { Maximal oxygen consumption } \\ \text { RPE } & \text { Rate of perceived exertion } \\ \text { WRDS } & \text { Waikato Regional Diabetes Service } \\ \mathrm{HbA1c} & \text { Glycated haemoglobin } \\ \text { Min } & \text { Minute/s } \\ \mathrm{m} & \text { Metre/s }\end{array}$

\section{References}

1. Stel G, Francescato MP, Stenner E, Geat M. Prolonged Exercise in Type 1 Diabetes: Performance of a Customizable Algorithm to Estimate the Carbohydrate Supplements to Minimize Glycemic Imbalances. PLoS One. 2015;10(4):e0125220.

2. WróBel M, Rokicka D, Czuba M, Gołaś A, Pyka $\measuredangle$, Greif M, et al. Aerobic as well as resistance exercises are good for patients with type 1 diabetes. Diabetes Research and Clinical Practice. 2018;144:93-101.

3. Hasan S, Shaw S, Gelling L, Kerr C, Meads C. Exercise modes and their association with hypoglycemia episodes in adults with type 1 diabetes mellitus: a systematic review. BMJ Open Diabetes Research \& Care. 2018;6(1):e000578.

4. Codella R, Terruzzi I, Luzi L. Why should people with type 1 diabetes exercise regularly? Acta Diabetologica. 2017;54(7):615-30.

5. García-García F, Kumareswaran K, Hovorka R, Hernando M. Quantifying the Acute Changes in Glucose with Exercise in Type 1 Diabetes: A Systematic Review and Meta-Analysis. Sports Medicine. 2015;45(4):587-99.

6. Samsum Diabetes Research Institute. ExCarbs. Available from https://excarbs.sansum.org/ [accessed May 2020] 2018 [

7. Gallen IW, Smart CE, Taplin CE, Adolfsson P, Lumb AN, Kowalski A, et al. Exercise management in type 1 diabetes: A consensus statement. The Lancet Diabetes \& Endocrinology. 2017;5(5):377-90.

8. Zaharieva D, Yavelberg L, Jamnik V, Cinar A, Turksoy K, Riddell MC. The effects of basal insulin suspension at the start of exercise on blood glucose levels during continuous versus circuit-based exercise in individuals with type 1 diabetes on continuous subcutaneous insulin infusion. Journal of Diabetes Technology and Therapeutics. 2017;19(6):370-8.

9. Riddell MC, Scott SN, Fournier PA, Colberg SR, Gallen IW, Moser O, et al. The competitive athlete with type 1 diabetes. Diabetologia. 2020;63(8):1475-90.

10. Grimm J, Ybarra J, Berné C, Muchnick S, Golay A. A new table for prevention of hypoglycaemia during physical activity in type 1 diabetic patients. Diabetes and Metabolism. 2004;30(5):465-70. 
11. Moser O, Mueller A, Eckstein ML, Ziko H, Aberer F, Treiber G, et al. Improved glycaemic variability and basal insulin dose reduction during a running competition in recreationally active adults with type 1 diabetes-A single-centre, prospective, controlled observational study. PloS one. 2020;15(9):e0239091.

12. Zaharieva DP, Riddell MC, Henske J. The Accuracy of Continuous Glucose Monitoring and Flash Glucose Monitoring During Aerobic Exercise in Type 1 Diabetes. Journal of Diabetes Science and Technology. 2019;13(1):140-1.

13. Dubé MC, Prud'homme D, Lemieux S, Lavoie C, Weisnagel SJ. Relation between energy intake and glycemic control in physically active young adults with type 1 diabetes. Journal of Science and Medicine in Sport. 2014;17(1):47-50.

14. Burr JF, Bredin SSD, Faktor MD, Warburton DER. The 6-Minute Walk Test as a Predictor of Objectively Measured Aerobic Fitness in Healthy Working-Aged Adults. The Physician and Sportsmedicine. 2011;39(2):133-9.

15. Ross RM, Murthy JN, Wollak ID, Jackson AS. The six minute walk test accurately estimates mean peak oxygen uptake. BMC Pulmonary Medicine. 2010;10(1):31.

16. Goodwin ML, Harris JE, Hernández A, Gladden LB. Blood lactate measurements and analysis during exercise: A guide for clinicians. Journal of Diabetes Science and Technology. 2007;1(4):558-69.

17. Williams N. The Borg Rating of Perceived Exertion (RPE) scale. Occupational Medicine. 2017;67(5):404-5.

18. Mauvais-Jarvis F, Sobngwi E, Porcher R, Garnier JP, Vexiau P, Duvallet A, et al. Glucose response to intense aerobic exercise in type 1 diabetes: maintenance of near euglycemia despite a drastic decrease in insulin dose. 2003;26(4):1316-7.

19. Riddell MC, Milliken J, therapeutics. Preventing exercise-induced hypoglycemia in type 1 diabetes using real-time continuous glucose monitoring and a new carbohydrate intake algorithm: an observational field study. Journal of Diabetes Technology. 2011;13(8):819-25.

20. Shugart C, Jackson J, Fields KB. Diabetes in sports. Journal of Sports Health. 2010;2(1):29-38.

\section{Figures}




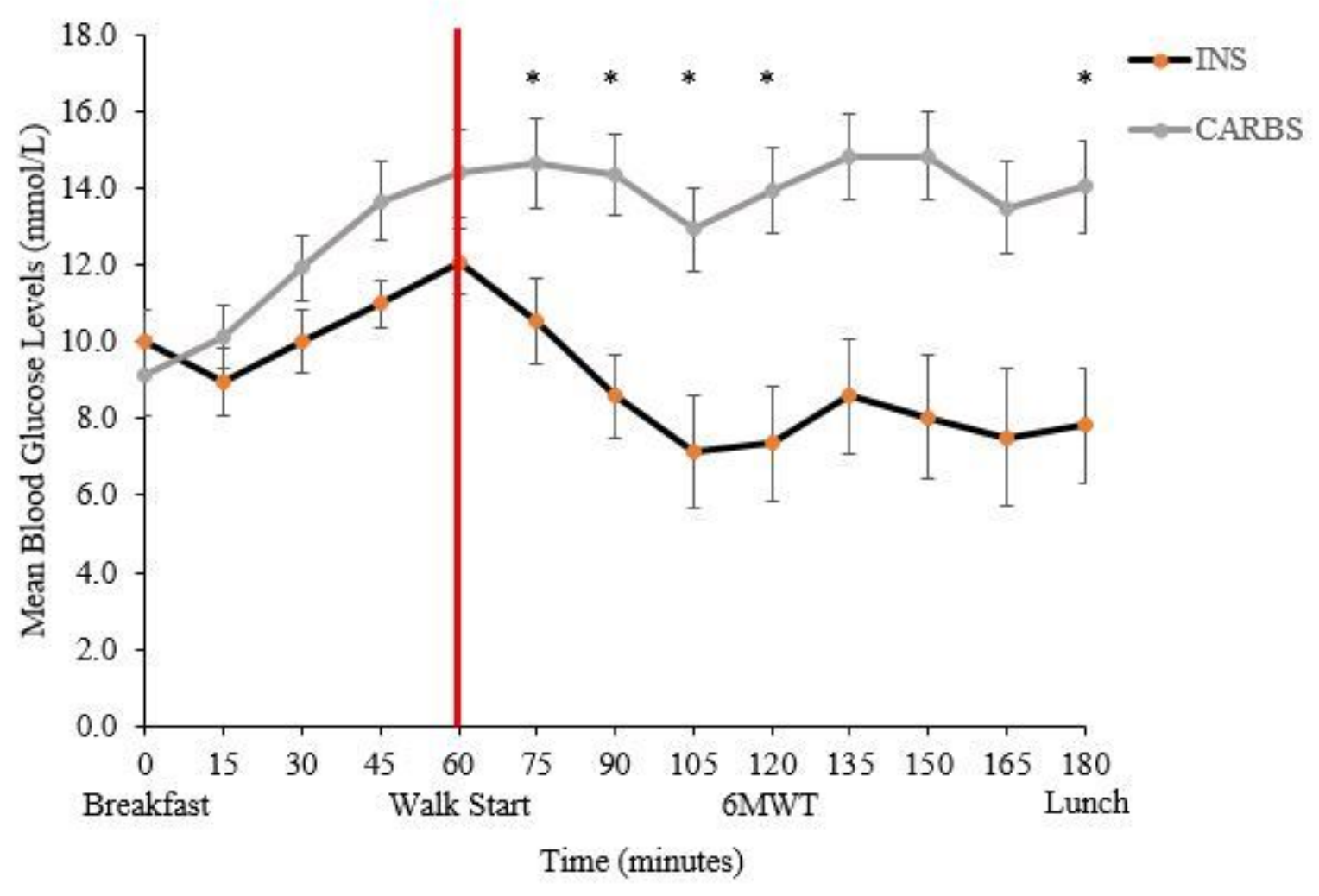

Figure 1

The effects of reducing bolus insulin with (INS) vs consuming carbs without insulin (CARBS) on blood glucose levels. ${ }^{*} \mathrm{P}<0.05$ between INS and CARBS 